\title{
Anagliptin increases insulin-induced skeletal muscle glucose uptake via an NO-dependent mechanism in mice
}

\author{
Hiroyuki Sato ${ }^{1} \cdot$ Naoto Kubota $^{1,2,3,4} \cdot$ Tetsuya Kubota $^{1,3,4,5} \cdot$ Iseki Takamoto $^{1}$ • \\ Kaito Iwayama ${ }^{6}$ - Kumpei Tokuyama ${ }^{6} \cdot$ Masao Moroi $^{5} \cdot$ Kaoru Sugi $^{5} \cdot K$ Keizo Nakaya ${ }^{7}$. \\ Moritaka Goto $^{7}$ - Takahito Jomori ${ }^{7}$. Takashi Kadowaki ${ }^{1}$
}

Received: 1 May 2016 / Accepted: 4 July 2016 / Published online: 15 August 2016

(C) Springer-Verlag Berlin Heidelberg 2016

\begin{abstract}
Aims/hypothesis Recently, incretin-related agents have been reported to attenuate insulin resistance in animal models, although the underlying mechanisms remain unclear. In this study, we investigated whether anagliptin, the dipeptidyl peptidase 4 (DPP-4) inhibitor, attenuates skeletal muscle insulin resistance through endothelial nitric oxide synthase (eNOS) activation in the endothelial cells. We used endothelium-specific Irs2-knockout (ETIrs2KO) mice, which show skeletal muscle insulin resistance resulting from a reduction of insulin-induced skeletal muscle capillary recruitment as a consequence of impaired eNOS activation. Methods In vivo, 8-week-old male ETIrs2KO mice were fed regular chow with or without $0.3 \%$ (wt/wt) DPP-4 inhibitor for 8 weeks to assess capillary recruitment and glucose uptake by the skeletal muscle. In vitro, human coronary arterial
\end{abstract}

endothelial cells (HCAECs) were used to explore the effect of glucagon-like peptide 1 (GLP-1) on eNOS activity.

Results Treatment with anagliptin ameliorated the impaired insulin-induced increase in capillary blood volume, interstitial insulin concentration and skeletal muscle glucose uptake in ETIrs2KO mice. This improvement in insulin-induced glucose uptake was almost completely abrogated by the GLP-1 receptor (GLP-1R) antagonist exendin-(9-39). Moreover, the increase in capillary blood volume with anagliptin treatment was also completely inhibited by the NOS inhibitor. GLP-1 augmented eNOS phosphorylation in HCAECs, with the effect completely disappearing after exposure to the protein kinase A (PKA) inhibitor H89. These data suggest that anagliptin treatment enhances insulin-induced capillary recruitment and interstitial insulin concentrations, resulting in improved skeletal muscle glucose

Hiroyuki Sato, Naoto Kubota and Tetsuya Kubota contributed equally to this work.

Electronic supplementary material The online version of this article (doi:10.1007/s00125-016-4071-8) contains peer-reviewed but unedited supplementary material, which is available to authorised users.

Naoto Kubota

nkubota-tky@umin.ac.jp

Takashi Kadowaki

kadowaki-3im@h.u-tokyo.ac.jp

1 Department of Diabetes and Metabolic Diseases, Graduate School of Medicine, The University of Tokyo, 7-3-1 Hongo, Bunkyo-ku, Tokyo 113-8655, Japan

2 Department of Clinical Nutrition Therapy, University of Tokyo, Tokyo, Japan
Clinical Nutrition Program, National Institute of Health and Nutrition, Tokyo, Japan

4 Laboratory for Metabolic Homeostasis, RIKEN Center for Integrative Medical Sciences, Kanagawa, Japan

5 Division of Cardiovascular Medicine, Toho University Ohashi Medical Center, Tokyo, Japan

6 Graduate School of Comprehensive Human Sciences, University of Tsukuba, Tsukuba, Japan

7 Mie Research Laboratories, Sanwa Kagaku Kenkyusho Co. Ltd, Mie, Japan 
uptake by directly acting on the endothelial cells via NO- and GLP-1-dependent mechanisms in vivo.

Conclusions/interpretation Anagliptin may be a promising agent to ameliorate skeletal muscle insulin resistance in obese patients with type 2 diabetes.

Keywords DPP-4 inhibitor · Endothelial cells · GLP-1 . Insulin resistance $\cdot$ Skeletal muscle glucose uptake

$\begin{array}{ll}\text { Abbreviations } \\ \text { DPP-4 } & \text { Dipeptidyl peptidase-4 } \\ \text { EGP } & \text { Endogenous glucose production } \\ \text { eNOS } & \text { Endothelial nitric oxide synthase } \\ \text { ETIrs2KO } & \text { Endothelium-specific Irs2-knockout } \\ \text { Ex-9-39 } & \text { Exendin-(9-39) } \\ \text { GIR } & \text { Glucose infusion rate } \\ \text { GLP-1 } & \text { Glucagon-like peptide 1 } \\ \text { GLP-1R } & \text { Glucagon-like peptide 1 receptor } \\ \text { HCAECs } & \text { Human coronary arterial endothelial cells } \\ \text { HF } & \text { High-fat } \\ \text { HCMVECs } & \text { Human cardiac microvascular endothelial cells } \\ \text { IR } \beta & \text { Insulin receptor } \beta \\ \text { L-NAME } & N^{\omega} \text {-nitro-L-arginine methyl ester } \\ \text { PKA } & \text { Protein kinase A } \\ \text { Rd } & \text { Rate of glucose disappearance }\end{array}$

\section{Introduction}

Skeletal muscle plays a pivotal role in insulin-induced glucose uptake, which is impaired in insulin-resistant states such as obesity and type 2 diabetes [1,2]. We previously demonstrated, using endothelium-specific Irs2-knockout (ETIrs2KO) mice, that the hyperinsulinaemia in high-fat (HF) diet-fed mice decreased the expression levels of Irs 2 in the endothelial cells, and this insulin receptor substrate 2 (IRS2) downregulation caused impaired insulin-induced capillary recruitment and insulin delivery, resulting in a reduction of skeletal muscle glucose uptake [3]. Moreover, beraprost sodium, a stable prostaglandin analogue, activated endothelial nitric oxide synthase (eNOS) independently of IRS2, and reversed the reduction in capillary blood volume/insulin-induced skeletal muscle glucose uptake in both ETIrs2KO and HF diet-fed mice [3]. Thus, agents inducing activation of eNOS in the endothelial cells may be promising candidates for amelioration of skeletal muscle insulin resistance.

Glucagon-like peptide 1 (GLP-1) receptor (GLP-1R) agonists improve fasting and postprandial glucose levels and pancreatic beta cell function, and are widely used in the treatment of type 2 diabetes mellitus [4-8]. In addition to these actions, GLP-1R agonists have been reported to attenuate insulin resistance in humans [9-11]. Recently, Chai et al demonstrated that a bolus injection of a GLP-1R agonist improved both the muscle microvascular blood volume and skeletal muscle glucose uptake in HF diet-fed rats [12]. Moreover, the GLP-1R agonist exendin-4 has been shown to enhance the phosphorylation of eNOS via the protein kinase A (PKA) pathway in endothelial cell lines [13]. These data suggest that GLP-1 may improve skeletal muscle glucose uptake through activating eNOS in the endothelial cells. As physiological GLP-1 is easily degraded by dipeptidyl peptidase-4 (DPP-4) [14, 15], DPP-4 inhibitors that induce GLP-1 activation have been used in the treatment of type 2 diabetes [16-21]. In addition to exerting actions mediated by GLP-1, DPP-4 inhibitors have been shown to inhibit cleavage of several non-incretin substrates, such as cytokines, chemokines and neuropeptides [22-24]; thus, DPP-4 inhibitors may have additional actions independent of those mediated by GLP-1. We therefore investigated whether the DPP-4 inhibitor anagliptin improved insulin-induced skeletal muscle glucose uptake through eNOS activation using ETIrs2KO mice.

\section{Methods}

Animals ETIrs2KO mice were generated as described previously [3], and housed under a $12 \mathrm{~h}$ light/dark cycle. The mice were fed regular chow, CE-2 (CLEA Japan, Tokyo, Japan), or CE-2 containing $0.3 \%$ (wt/wt) anagliptin, for 8 weeks. Anagliptin provided by Sanwa Kagaku Kenkyusho (Nagoya, Japan) was used as the DPP-4 inhibitor [25]. Male ETIrs $2 \mathrm{KO}$ mice, 8 weeks old, were assigned to receive anagliptin or not, and $\operatorname{Irs} 2^{\text {flox/flox }}$ mice were used as the control. The animal care and experimental procedures used in this study were accepted by the Animal Care Committee of the University of Tokyo.

Measurement of the plasma variables Plasma anagliptin levels were measured with a validated liquid chromatography/ tandem MS (LC-MS/MS) assay, as described previously [26]. Plasma DPP-4 activity was determined by a fluorometric assay using Gly-Pro-MCA (Peptide Institute, Osaka, Japan), slightly modified from a previously described protocol [27]. Further information is provided in the electronic supplementary material (ESM) Methods.

Insulin tolerance test Mice were intraperitoneally administered with human insulin (Humulin R; Eli Lilly Japan, Kobe, Japan) at $0.75 \mathrm{U} / \mathrm{kg}$ body weight after $1 \mathrm{~h}$ of fasting. Blood glucose levels were measured at $0,20,40,60,80,100$ and $120 \mathrm{~min}$ after insulin administration using an automatic glucometer (Sanwa Kagaku Kenkyusho). 
Hyperinsulinaemic-euglycaemic clamp Clamp studies were performed as described previously [28], with some modifications. Briefly, 2 days before the study, an infusion catheter was inserted into the right jugular vein under general anaesthesia. Studies were conducted under conscious and unstressed conditions after $3 \mathrm{~h}$ of denying the animals access to food. Insulin was continuously administered $\left(5.0 \mathrm{mU} \mathrm{kg}^{-1} \mathrm{~min}^{-1}\right)$, and the blood glucose concentration, monitored every $5 \mathrm{~min}$, was maintained at approximately $6.67 \mathrm{mmol} / \mathrm{l}$ by the infusion of glucose $(5 \mathrm{~g}$ of glucose $/ 10 \mathrm{ml}$ enriched to about $20 \%$ [wt/ vol.] with $\left[6,6-{ }^{2} \mathrm{H}_{2}\right]$ glucose [Sigma-Aldrich, Tokyo, Japan]) for $60 \mathrm{~min}$. Blood samples were obtained via tail-tip bleeds at 30,45 and $60 \mathrm{~min}$ to determine the rate of glucose disappearance (Rd). Rd was calculated according to nonsteady-state equations, and endogenous glucose production (EGP) was calculated as the difference between the $\mathrm{Rd}$ and the exogenous glucose infusion rate (GIR).

Capillary blood volume The capillary blood volume was determined using contrast-enhanced ultrasound, by slight modification of a protocol described previously [29]. In brief, the hindlimb muscles were imaged in the short axis using an ultrasound system equipped with a $40 \mathrm{MHz}$ transducer (RMV 704) (Vevo 770; VisualSonics, Toronto, ON, Canada). Infusion of Sonazoid (Daiichi Sankyo, Tokyo, Japan) was administered to the animals, which were divided into two groups for the measurements at 0 and $60 \mathrm{~min}$ after the hyperinsulinaemic-euglycaemic clamp. A high-power ultrasound with a frequency of $1 \mathrm{MHz}$ was applied to the lower leg muscles, and images were obtained for $30 \mathrm{~s}$ to assess the enhancement. The ultrasound intensity in decibels within the region of interest was converted to acoustic intensity after background subtraction of the $0.5 \mathrm{~s}$ ultrasound images, using the formula of microvascular volume, fill rate constant and capillary blood volume was $y=\mathrm{A}\left(1-\mathrm{e}^{-\beta t}\right)$, where $y$ is capillary blood volume, A is plateau video intensity, and $t$ is the pulsing interval.

\section{Interstitial concentrations of insulin in the skeletal muscle} Muscle microdialysis was conducted in the hindlimb muscles using a $4 \mathrm{~mm}$ microdialysis tube (CMA-20; CMA Microdialysis, Stockholm, Sweden), at a rate $0.3 \mu \mathrm{l} / \mathrm{min}$, as described previously [30]. We carried out calibration using the no-net-flux technique. In brief, four known concentrations of insulin $(0 \mathrm{pmol} / 1,8.75 \mathrm{pmol} / \mathrm{l}, 17.5 \mathrm{pmol} / 1$ and $26.25 \mathrm{pmol} / \mathrm{l})$ above and below the expected concentration in the skeletal muscle were selected. The insulin solutions were added to the perfusate and the net changes in the concentrations of the analytes in the dialysate were archived insulin $_{\text {out }}-$ insulin $_{\text {in }}=$ net change). According to regression analysis, a linear relationship was obtained between the concentrations in the perfusates and the dialysates. The intercept with the $x$-axis shows the insulin concentrations in the perfusate at equilibrium with the surrounding medium, and the slope of the line exhibits the dialysis recovery by the no-net-flux technique. The insulin concentrations in the interstitial fluid were determined from the dialysis recovery by the no-net-flux technique and in vivo dialysate insulin concentration, as described previously [31].

Insulin signalling in the skeletal muscle in vivo and ex vivo To investigate insulin signalling in the skeletal muscle, insulin (105 pmol/l) was injected via the inferior vena cava. The soleus and gastrocnemius muscles were dissected $60 \mathrm{~min}$ after the insulin infusion and immediately frozen in liquid nitrogen. Insulin signalling in the isolated skeletal muscle was carried out as described previously [32], with slight modifications. In brief, the soleus and gastrocnemius muscles were dissected out, cut into strips of 10-15 mg, and incubated for $10 \mathrm{~min}$ at $37^{\circ} \mathrm{C}$ in $1 \% \mathrm{BSA} / \mathrm{DMEM}$ with or without $100 \mathrm{nmol} / \mathrm{l}$ insulin. The muscles were rapidly washed twice with PBS buffer to stop the reaction. For the western blot analysis, the muscle pieces were immediately frozen in liquid nitrogen. Information about western blot analysis and antibodies is provided in ESM Methods.

Administration of exendin-(9-39) Exendin-(9-39) (Ex-9-39) (Sigma-Aldrich) was administered as described previously [33], with a slight modification of the method. In brief, Ex-9-39 or vehicle $(0.9 \%$ [wt/vol.] NaCl, $1 \%$ [wt/vol.] BSA) was administered subcutaneously at a rate of $50 \mathrm{pmol} \mathrm{kg}^{-1} \mathrm{~min}^{-1}$ using mini-osmotic pumps (model 1002; DURECT Corporation, Cupertino, CA, USA), which were replaced every 2 weeks. Ex-9-39 was administered for 8 weeks.

Cell culture Human coronary arterial endothelial cells (HCAECs) and human cardiac microvascular endothelial cells (HCMVECs) were purchased from Lonza Japan (Tokyo, Japan) and maintained at $37^{\circ} \mathrm{C}$ under $5 \% \mathrm{CO}_{2}$ in endothelial growth medium-2 (Lonza Japan) supplemented with hydrocortisone, human epidermal growth factor (hEGF), FBS, vascular endothelial growth factor (VEGF), human fibroblast growth factor (hFGF)-B, R3 insulin-like growth factor (IGF)-1, ascorbic acid and gentamicin/amphotericin B. The HCAECs and HCMVECs were grown to confluence, then incubated overnight under serum starvation. H89 or vehicle was added $30 \mathrm{~min}$ prior to the GLP-1 stimulation. Insulin and 5-aminoimidazole-4-carboxamide ribonucleotide (AICAR) were incubated for $30 \mathrm{~min}$. All experiments were performed with cells at passage 5-10.

NOS inhibition Mice were infused with either insulin or insulin with a superimposed infusion of $N^{\omega}$-nitro-L-arginine methyl ester (L-NAME) $(50 \mathrm{mg} / \mathrm{kg}$ bolus followed by continuous infusion at $10 \mathrm{mg} \mathrm{kg}^{-1} \mathrm{~h}^{-1}$ ) during the 
hyperinsulinaemic-euglycaemic clamp study. We measured the capillary blood volume at $60 \mathrm{~min}$ after the infusion.

RNA preparation and RT-PCR Total RNA was prepared from the tissues and cell lines using the Trizol reagent (Thermo Fisher Scientific, Yokohama, Japan) and RNeasy Mini Kit (Qiagen Sciences, Germantown, MD, USA), respectively, according to the manufacturer's instructions. RNA, $1 \mu \mathrm{g}$, was used for generating cDNA using random hexamers with MultiScribe reverse transcription reagents (Thermo Fisher Scientific). In the tissue samples, TaqMan quantitative PCR $\left(50^{\circ} \mathrm{C}\right.$ for $2 \mathrm{~min}, 95^{\circ} \mathrm{C}$ for $10 \mathrm{~min}$, followed by 40 cycles of $95^{\circ} \mathrm{C}$ for $15 \mathrm{~s}, 60^{\circ} \mathrm{C}$ for $1 \mathrm{~min}$ ) was then performed with ABI Prism 7900 PCR (Thermo Fisher Scientific) to amplify $\operatorname{Tnf} \alpha$ (also known as Tnf), Mcp 1 (also known as $C c l 2$ ), resistin, $R b p 4, \operatorname{Veg} f$ (also known as $V e g f a)$, Gapdh and $\beta$-actin cDNA. The expression levels of each of the transcripts were normalised to the constitutive expression levels of Gapdh and $\beta$-actin mRNA. In the cell lines, RT-PCR was performed in a DNA thermal cycler for 40 cycles $\left(1 \mathrm{~min}\right.$ at $94^{\circ} \mathrm{C}, 30 \mathrm{~s}$ at $56^{\circ} \mathrm{C}$, and $1 \mathrm{~min}$ at $72^{\circ} \mathrm{C}$ ). Information about TaqMan probes and Glp $1 r$ specific primers is provided in ESM Methods.

Statistical analysis Results are expressed as means \pm SEM. The statistical significance of all possible pairwise differences in the means were determined by one-way ANOVA followed by post hoc Tukey-Kramer test. The statistical significance level was set at $p<0.05$.

\section{Results}

Treatment with anagliptin ameliorated impaired insulininduced skeletal muscle glucose uptake in ETIrs2KO mice When anagliptin was administrated to ETIrs2 $\mathrm{KO}$ mice, plasma anagliptin levels were increased by about $1200 \mathrm{ng} / \mathrm{ml}$ (ESM Fig. 1), similar to those reported for healthy volunteers receiving anagliptin [34]. Plasma DPP-4 activity was inhibited by approximately $90 \%$ in the anagliptin-treated ETIrs $2 \mathrm{KO}$ mice (Fig. 1a). However, plasma levels of active GLP-1 were markedly increased in the anagliptin-treated ETIrs2KO mice (Fig. 1b). There were no significant differences in the body weight gain or food intake among the three groups (Fig. 1c, d). These data suggest that anagliptin treatment increased the active GLP-1 levels in ETIrs2KO mice without inducing any changes in body weight or food intake.

To investigate the effect of anagliptin on the insulin resistance observed in the ETIrs2 $\mathrm{KO}$ mice, an insulin tolerance test was performed. Although the hypoglycaemic response to insulin was lower in the ETIrs2KO mice than in the control mice, as reported previously by us [3], it did not differ between the control and anagliptin-treated ETIrs $2 \mathrm{KO}$
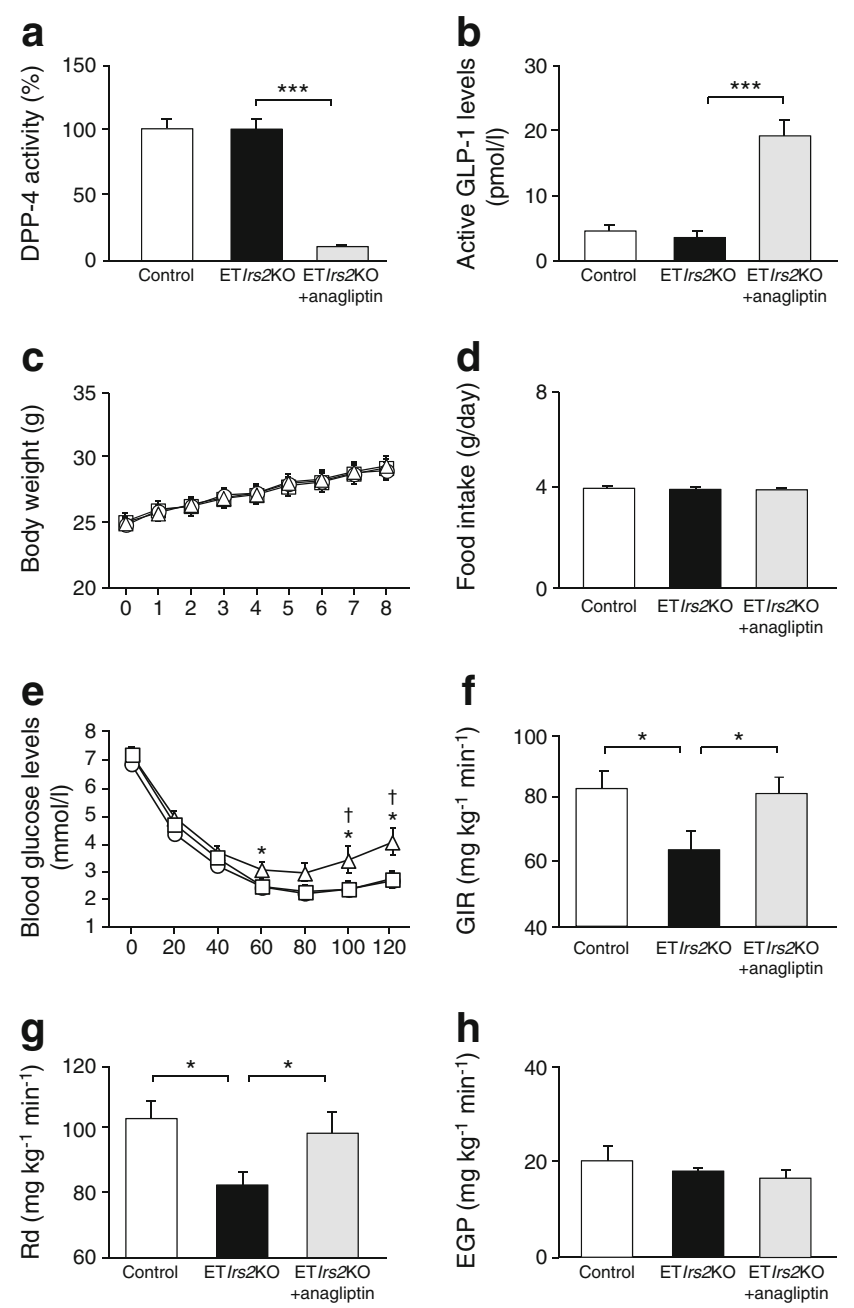

Fig. 1 Treatment with anagliptin restored the impaired insulin-induced skeletal muscle glucose uptake in the ETIrs2KO mice. Plasma DPP-4 activity (a) and active GLP-1 levels (b) in the control, ETIrs2KO and anagliptin-treated ETIrs2KO mice $(n=21-24)$. Values are the means \pm SEM. $* * * p<0.001$, ETIrs $2 \mathrm{KO}$ mice vs anagliptin-treated ETIrs $2 \mathrm{KO}$ mice. (c) Body weight in the control (white circles), ETIrs2KO (white triangles) and anagliptin-treated ETIrs2KO (white squares) mice $(n=15)$. (d) Food intake in the control, ETIrs $2 \mathrm{KO}$ and anagliptin-treated ETIrs2KO mice $(n=7)$. (e) Blood glucose levels during the insulin tolerance test in the control (white circles), ETIrs2KO (white triangles) and anagliptin-treated ETIrs2KO (white squares) mice $(n=13-15)$. Values are the means \pm SEM. $* p<0.05$, control mice vs ETIrs $2 \mathrm{KO}$ mice; ${ }^{\dagger} p<0.05$, ETIrs $2 \mathrm{KO}$ mice vs anagliptin-treated ETIrs2KO mice. GIR (f), Rd (g) and EGP (h) during the hyperinsulinaemic-euglycaemic clamp study in the control, ETIrs2 $\mathrm{KO}$ and anagliptin-treated ETIrs $2 \mathrm{KO}$ mice $(n=7-9)$. Values are the means \pm SEM. $* p<0.05$ vs ETIrs $2 \mathrm{KO}$ mice

mice (Fig. 1e). These results suggest that anagliptin treatment ameliorated the insulin resistance in the ETIrs $2 \mathrm{KO}$ mice. Consistent with these data, the decrease in GIR in the ETIrs $2 \mathrm{KO}$ mice was completely reversed by anagliptin treatment during the hyperinsulinaemic-euglycaemic clamp study (Fig. 1f). Moreover, anagliptin treatment also increased Rd in the ETIrs2KO mice to a level similar to that observed in the control mice (Fig. 1g). However, there were no significant 
differences in EGP among the three groups (Fig. 1h). These data suggest that anagliptin treatment ameliorated the impaired insulin-induced skeletal muscle glucose uptake in the ETIrs2KO mice. No significant differences were observed in the rectal temperature, plasma lipid or adipocytokine profiles, or mRNA expression levels of inflammatory cytokines and adipocytokines among the three groups (ESM Fig. 2a-m).

Anagliptin ameliorated the decreased insulin-induced capillary blood volume and interstitial insulin concentrations in ETIrs2KO mice To delineate the mechanism underlying the improvement in insulin-induced skeletal muscle glucose uptake in the anagliptin-treated ETIrs $2 \mathrm{KO}$ mice, the capillary blood volume was measured in the three groups. Treatment with anagliptin restored the decreased insulin-induced capillary blood volume in the ETIrs $2 \mathrm{KO}$ mice to a level similar to that observed in the control mice (Fig. 2a, ESM Video 1a-c). Moreover, the reduced insulin-induced interstitial concentration of insulin observed in the ETIrs $2 \mathrm{KO}$ mice was completely restored in the anagliptintreated ETIrs2KO mice, even though no significant differences were observed in the plasma insulin levels among the three groups during the hyperinsulinaemic-euglycaemic clamp study (Fig. 2b, c). These data suggest that anagliptin treatment ameliorated the decreased insulin-induced capillary blood volume and interstitial insulin concentrations in the ETIrs2KO mice. Consistent with the results for the interstitial insulin concentrations, the insulin-induced phosphorylation levels of insulin receptor $\beta(\operatorname{IR} \beta)$ and Akt in the skeletal muscle at $60 \mathrm{~min}$ after insulin infusion into the inferior vena cava were also significantly restored in the anagliptin-treated ETIrs2KO mice (Fig. 2d, e). However, in the isolated skeletal muscle, the phosphorylation levels of IR $\beta$ and Akt did not differ between the control and ETIrs $2 \mathrm{KO}$ mice, and no significant differences in the phosphorylation levels between the anagliptin-treated and non-anagliptin-treated ETIrs $2 \mathrm{KO}$ mice were observed (Fig. 2f, g). These data suggest that the improved insulin delivery induced by anagliptin treatment ameliorated impaired insulin signalling in the skeletal muscle. Taken together, anagliptin treatment restored the decreased insulin-induced capillary blood volume and interstitial insulin concentrations, resulting in amelioration of the impaired insulin signalling in the skeletal muscle of the ETIrs $2 \mathrm{KO}$ mice.

\section{Treatment with anagliptin increased insulin-induced} skeletal muscle glucose uptake in the ETIrs2KO mice via a GLP-1-dependent pathway To address the extent to which GLP-1 activation by anagliptin is involved in the improvement of the insulin-induced skeletal muscle glucose uptake in ETIrs2KO mice, Ex-9-39, an antagonist of GLP-1R, was administered to the anagliptin-treated ETIrs $2 \mathrm{KO}$ mice. The improvement in GIR and Rd in the anagliptin-treated ETIrs $2 \mathrm{KO}$ mice was significantly abrogated by treatment with
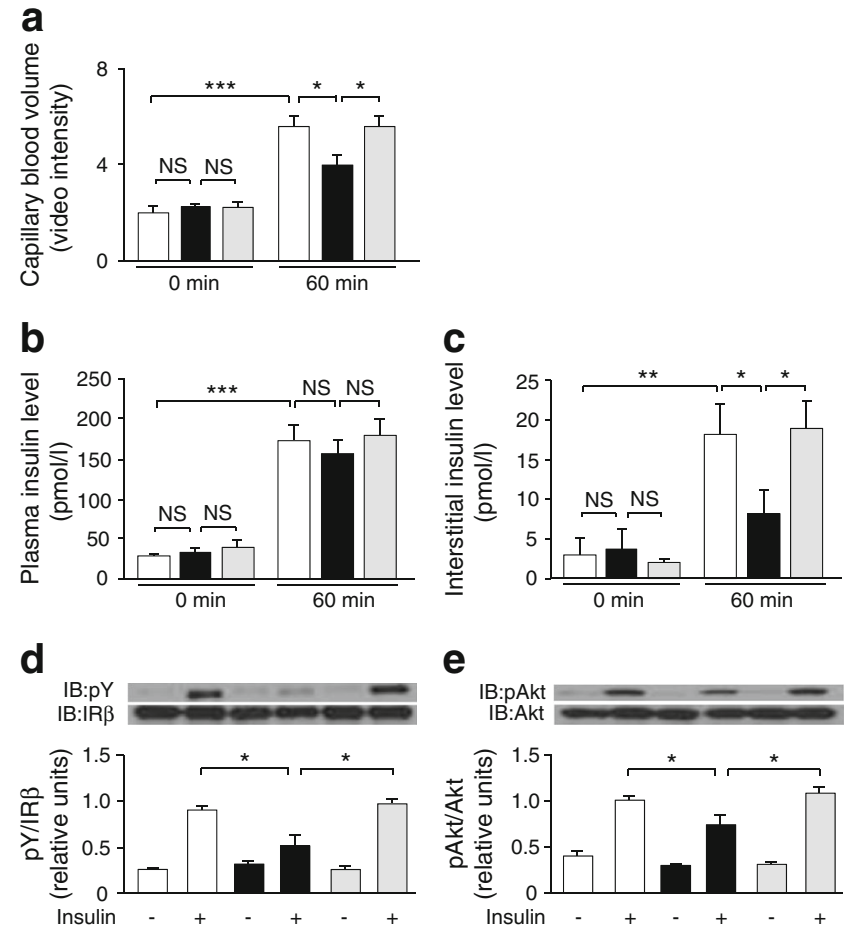

f
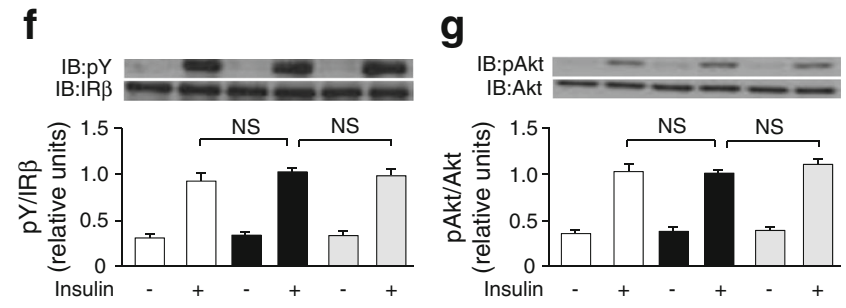

Fig. 2 Treatment with anagliptin restored the insulin-induced increase in capillary blood volume and interstitial concentration of insulin in the ETIrs2KO mice. Capillary blood volume (a), and plasma (b) and interstitial (c) insulin levels during the hyperinsulinaemic-euglycaemic clamp study in the control (white bars), ETIrs2KO (black bars) and anagliptin-treated ETIrs2KO (grey bars) mice $(n=9-12)$. Values are the means \pm SEM. $* p<0.05$ vs ETIrs $2 \mathrm{KO}$ mice; $* * p<0.01$ and $* * * p<0.001,0$ min vs 60 min. Phosphorylation levels of IR $\beta$ (d) and Akt (e) in the skeletal muscle of control (white bars), ETIrs2KO (black bars) and anagliptin-treated ETIrs $2 \mathrm{KO}$ (grey bars) mice at $60 \mathrm{~min}$ after insulin infusion $(n=4)$. $* p<0.05$ vs ETIrs $2 \mathrm{KO}$ mice. Insulin-stimulated phosphorylation levels of $\operatorname{IR} \beta$ (f) and Akt (g) in the isolated skeletal muscle of control (white bars), ETIrs2KO (black bars) and anagliptintreated ETIrs2KO (grey bars) mice $(n=4)$. Values are the means \pm SEM. $\mathrm{IB}$, immunoblot; $\mathrm{pY}$, phosphorylated tyrosine

Ex-9-39 in the hyperinsulinaemic-euglycaemic clamp study (Fig. 3a, b). However, no significant difference in EGP was found among the four groups (Fig. 3c). These data suggest that anagliptin treatment improved insulin-induced skeletal muscle glucose uptake in the ETIrs2KO mice via a GLP-1-dependent pathway.

Treatment with L-NAME completely abrogated the insulin-induced increase in capillary blood volume in anagliptin-treated ETIrs2KO mice To determine whether the improvement in capillary blood volume in the anagliptin- 
a

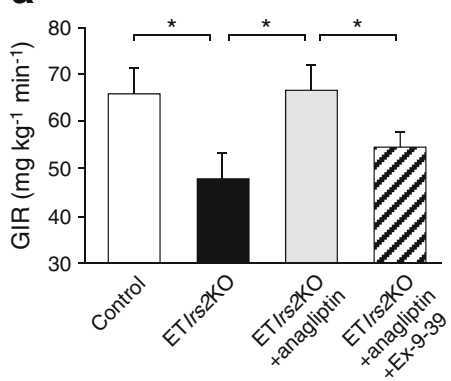

Fig. 3 GLP-1 activation by anagliptin treatment mainly restored the insulin-induced skeletal muscle glucose uptake in ETIrs2KO mice. GIR (a), Rd (b) and EGP (c) in the anagliptin-treated ETIrs2KO mice b

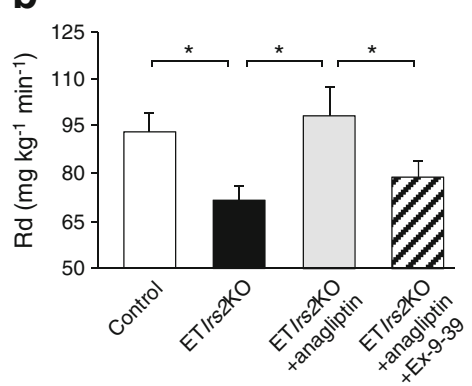

C

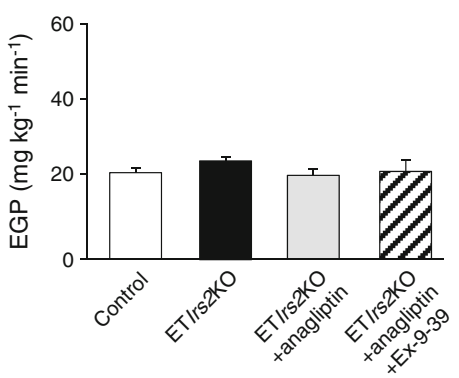

following Ex-9-39 treatment in the hyperinsulinaemic-euglycaemic clamp study $(n=8-11)$. Values are the means \pm SEM. $* p<0.05$

a

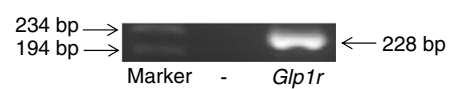
treated ETIrs2KO mice. Although there were no significant differences among the six groups in the capillary blood volume before insulin infusion, treatment with L-NAME completely reversed the insulin-induced increase in capillary blood volume in the control mice. This result suggests that the insulin-induced increase in capillary blood volume in the control mice was mediated by an NO-dependent mechanism (Fig. 4). Similarly, the insulin-induced increase in capillary blood volume in the anagliptin-treated ETIrs2KO mice was completely abrogated by treatment with L-NAME. These data suggest that anagliptin treatment increased the insulin-induced capillary blood volume via an NO-dependent pathway in ETIrs2KO mice.

\section{GLP-1 elevated the phosphorylation levels of eNOS via the} PKA pathway in endothelial cells The expression levels of Glp1r mRNA were confirmed in HCAECs (Fig. 5a). To investigate whether GLP-1 activated eNOS in the HCAECs, the protein and phosphorylation levels of eNOS were measured after GLP-1 stimulation in these cells. Although

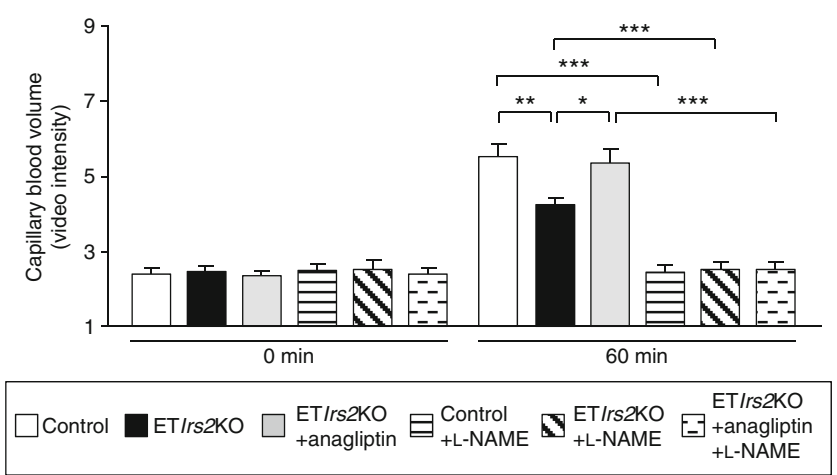

Fig. 4 Treatment with L-NAME completely abrogated the insulin-induced increase in capillary blood volume in the anagliptin-treated ETIrs2KO mice. Capillary blood volume in the anagliptin-treated ETIrs $2 \mathrm{KO}$ mice following L-NAME treatment $(n=8-12)$. Values are the means \pm SEM. $* p<0.05, * * p<0.01$ and $* * * p<0.001$

(1)
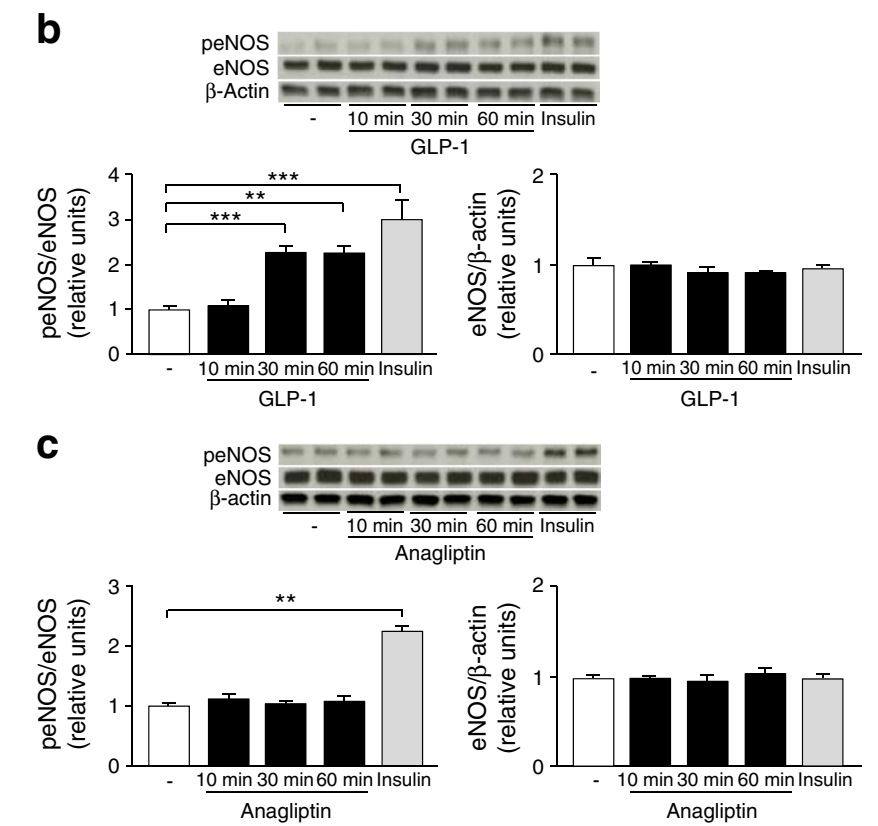

d
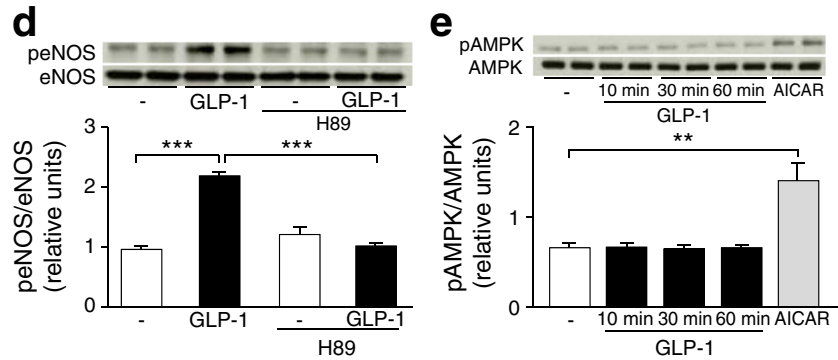

Fig. 5 GLP-1 stimulated phosphorylation of eNOS via the PKA pathway in HCAECs. (a) Glp1r expression in HCAECs using RT-PCR $(n=3)$. $(\mathbf{b}, \mathbf{c})$ GLP-1- and anagliptin-stimulated phosphorylation levels of eNOS in HCAECs $(n=4-6)$. Values are the means \pm SEM. $* * p<0.01$ and $* * * p<0.001$ vs untreated condition. (d) GLP-1-stimulated phosphorylation levels of eNOS following treatment with H89, an inhibitor of PKA, in HCAECs $(n=4-6)$. (e) GLP-1-stimulated phosphorylation levels of AMPK in HCAECs $(n=4-6)$. Values are the means \pm SEM. $* * p<0.01$ and $* * * p<0.001$ 
the levels of eNOS protein did not differ significantly before and after GLP-1 stimulation, the eNOS phosphorylation levels were significantly increased 30 and 60 min after GLP-1 stimulation (Fig. 5b). However, the protein and phosphorylation levels of eNOS did not differ significantly before or after anagliptin treatment (Fig. 5c). These data suggest that GLP-1, and not directly anagliptin, activates eNOS in the endothelial cells. Moreover, in the presence of the PKA inhibitor H89, the GLP-1-stimulated eNOS phosphorylation was completely abolished (Fig. 5d). However, GLP-1 stimulation had no significant effect on AMPK phosphorylation levels (Fig. 5e). Just as in the case of the HCAECs, levels of Glp1r were confirmed in the HCMVECs (ESM Fig. 3a). While there was no change in the phosphorylation level of eNOS associated with anagliptin administration, the phosphorylation level of eNOS was significantly increased after GLP-1 stimulation (ESM Fig. 3b). Moreover, in the presence of the PKA inhibitor H89, the GLP-1-stimulated eNOS phosphorylation was almost completely abolished (ESM Fig. 3b). These results suggest that GLP-1 activates eNOS via the PKA pathway in endothelial cells.

\section{Discussion}

In this study, we demonstrated that anagliptin treatment enhanced insulin-induced capillary recruitment and interstitial insulin concentrations, resulting in improvement of skeletal muscle glucose uptake in ETIrs2 $2 \mathrm{KO}$ mice. These effects were abrogated by treatment with a GLP-1 antagonist. Moreover, pre-treatment with L-NAME also significantly attenuated the insulin-induced capillary recruitment in anagliptin-treated ETIrs2KO mice. These data suggest that anagliptin treatment enhances the insulin-induced capillary recruitment and interstitial insulin concentrations, resulting in improved skeletal muscle glucose uptake in vivo via direct actions on the endothelial cells through GLP-1- and NO-dependent pathways.

As no effective antibodies to GLP-1R are currently available [35], the protein levels of GLP-1R could not be assessed in endothelial cells. However, the expression of Glp1r mRNA in HCAECs has been confirmed in a previous study [36]. GLP-1 has been shown to increase the plasma NO levels and improve endothelial-dependent vascular relaxation [37]. These data suggest that functional GLP-1R is expressed in endothelial cells. In the current study, although anagliptin treatment did not change the phosphorylation level of eNOS, eNOS phosphorylation was increased by GLP-1 via the PKA pathway in HCAECs. Consistent with our results, Matsubara et al reported that while DPP-4 inhibitors did not change the phosphorylation levels of eNOS, treatment with GLP-1 or GLP-1 plus DPP-4 inhibitor significantly increased the eNOS phosphorylation level in HCAECs [36]. These data suggest that anagliptin stimulates eNOS activity by a GLP-1-dependent mechanism, potentially via PKA-eNOS phosphorylation.

Nakagami et al previously demonstrated using a spontaneously hypertensive corpulent congenic (SHR)/NDmcr-cp rat model that DPP-4 inhibitors improved endothelial function [38]. This rat model, a spontaneously hypertensive strain with a nonsense mutation in the leptin receptor, shows obesity, severe hypertension, insulin resistance, hyperglycaemia, increased levels of inflammatory cytokines and hyperlipidaemia. In the aforementioned study, DPP-4 inhibitors ameliorated metabolic abnormalities such as glucose intolerance and inflammatory cytokines, as well as endothelial function. Thus, it was difficult to determine whether the DPP-4 inhibitor improved endothelial function via a direct effect or via indirect effects such as improvement in the risk factor profile. In contrast, ETIrs2 $2 \mathrm{KO}$ mice do not show any obesity or changes in risk factor profile, including levels of triacylglycerol, FFA, leptin, inflammatory cytokines and adipocytokines, on a normal diet, despite also exhibiting endothelial dysfunction (ESM Fig. 2a-m). Moreover, the body weight, risk factor profile and levels of inflammatory cytokines and adipocytokines remained unaltered by anagliptin treatment in the ETIrs $2 \mathrm{KO}$ mice (ESM Fig. 2a-m). Thus, ETIrs2KO mice are thought to be appropriate as a model for investigating the effects of DPP-4 inhibitors.

We demonstrated that eNOS phosphorylation level was significantly increased from $30 \mathrm{~min}$ after GLP-1 stimulation in vitro (Fig. 5b). This activation of eNOS was completely abolished in the presence of the PKA inhibitor, H89. Dong et al reported that muscle microvascular recruitment induced by bolus infusion of GLP-1 was inhibited by the administration of H89 [39]. These findings suggest that anagliptin probably improves skeletal muscle capillary recruitment via the PKA pathway in mice. However, to investigate whether anagliptin indeed regulates skeletal muscle capillary recruitment via the PKA pathway in vivo, further study is needed using endothelial cell-specific $P k a$-knockout mice ( $P k a$ is also known as Prkaca).

Although it has been demonstrated that bolus injection of a GLP-1R agonist improved muscle microvascular blood volume and skeletal muscle glucose uptake in HF diet-fed rats [12], the causal relationship between increased muscle microvascular blood volume and amelioration of skeletal glucose uptake is yet to be clarified. This study is the first to demonstrate this causal relationship using ETIrs2KO mice; we showed that chronic treatment with anagliptin enhanced insulin-induced capillary recruitment and interstitial insulin concentrations, thereby improving the skeletal muscle glucose uptake in vivo, by directly acting on endothelial cells via NO- and GLP-1-dependent mechanisms. The findings of our study may be of clinical relevance, as GLP-1R agonists and DPP4 inhibitors have been reported to attenuate insulin 
resistance in humans $[9-11,40]$. Taken together, these data suggest that anagliptin might be a promising agent to improve skeletal muscle insulin resistance in obese patients with type 2 diabetes.

Acknowledgements We thank E. Hirata, A. Nagano, Y. Okonogi and M. Henmi (Department of Diabetes and Metabolic Diseases, Graduate School of Medicine, University of Tokyo, Tokyo, Japan) for their excellent technical assistance and assistance with the animal care.

Funding This work was supported by a grant for Translational Systems Biology and Medicine Initiative (TSBMI) from the Ministry of Education, Culture, Sports, Science and Technology of Japan, a Grant-in-aid for Scientific Research in Priority Areas (A) (16209030), (A) (18209033) and (S) (20229008) from the Ministry of Education, Culture, Sports, Science and Technology of Japan (to TKa), and a Grant-in-aid for Scientific Research in Priority Areas (C) (19591037) and (B) (21390279) from the Ministry of Education, Culture, Sports, Science, and Technology of Japan (to NK).

Duality of interest The authors declare that there is no duality of interest associated with this manuscript.

Contribution statement $\mathrm{HS}, \mathrm{NK}, \mathrm{TKu}$ and TKa were the substantial contributors in terms of study conception and design, acquisition and interpretation of data and writing the manuscript. IT made substantial contributions to the conceptual design and drafting the manuscript. KI and KT contributed substantially to the acquisition of data and revising the manuscript. MM and KS contributed substantially to the interpretation of data and revising the manuscript. $\mathrm{KN}, \mathrm{MG}$ and $\mathrm{TJ}$ made substantial contributions to the conceptual design and drafting the manuscript. TKa is the guarantor of this work, and as such, had full access to all the data in the study and takes responsibility for the integrity of the data and accuracy of the data analysis. All authors read and approved the published version of this manuscript.

\section{References}

1. DeFronzo RA (1988) Lilly lecture, 1987. The triumvirate: beta-cell, muscle, liver. A collusion responsible for NIDDM. Diabetes 37: 667-687

2. Barrett EJ, Eggleston EM, Inyard AC et al (2009) The vascular actions of insulin control its delivery to muscle and regulate the rate-limiting step in skeletal muscle insulin action. Diabetologia 52:752-764

3. Kubota T, Kubota N, Kumagai H et al (2011) Impaired insulin signaling in endothelial cells reduces insulin-induced glucose uptake by skeletal muscle. Cell Metab 13:294-307

4. Nauck M (2015) Incretin therapies: highlighting common features and differences in the modes of action of GLP-1 receptor agonists and DPP-4 inhibitors. Diabetes Obes Metab 18:203-216

5. Nauck M, Frid A, Hermansen K et al (2009) Efficacy and safety comparison of liraglutide, glimepiride, and placebo, all in combination with metformin, in type 2 diabetes. Diabetes Care 32:84-90

6. Buse JB, Rosenstock J, Sesti G et al (2009) Liraglutide once a day versus exenatide twice a day for type 2 diabetes: a 26 -week randomised, parallel-group, multinational, open-label trial (LEAD-6). Lancet 374:39-47
7. Divino V, DeKoven M, Hallinan S et al (2014) Glucagon-like peptide-1 receptor agonist treatment patterns among type 2 diabetes patients in six European countries. Diabetes Ther 5:499-520

8. Usui R, Yabe D, Kuwata H, Murotani K, Kurose T, Seino Y (2015) Retrospective analysis of safety and efficacy of liraglutide monotherapy and sulfonylurea-combination therapy in Japanese type 2 diabetes: association of remaining beta cell function and achievement of $\mathrm{HbA} 1 \mathrm{c}$ target one year after initiation. J Diabet Complications 29:1203-1210

9. Diaz-Soto G, de Luis DA, Conde-Vicente R, Izaola-Jauregui O, Ramos C, Romero E (2014) Beneficial effects of liraglutide on adipocytokines, insulin sensitivity parameters and cardiovascular risk biomarkers in patients with Type 2 diabetes: a prospective study. Diabetes Res Clin Pract 104:92-96

10. Kim SH, Abbasi F, Lamendola C et al (2013) Benefits of liraglutide treatment in overweight and obese older individuals with prediabetes. Diabetes Care 36:3276-3282

11. Garber A, Henry R, Ratner R et al (2009) Liraglutide versus glimepiride monotherapy for type 2 diabetes (LEAD-3 Mono): a randomized, 52 week, phase 3, double-blind, parallel treatment trial. Lancet 373:473-481

12. Chai W, Zhang X, Barrett EJ, Liu Z (2014) Glucagon-like peptide 1 recruits muscle microvasculature and improves insulin's metabolic action in the presence of insulin resistance. Diabetes 63:2788-2799

13. Erdogdu O, Nathanson D, Sjoholm A, Nystrom T, Zhang Q (2010) Exendin-4 stimulates proliferation of human coronary artery endothelial cells through eNOS-, PKA- and PI3K/Akt-dependent pathways and requires GLP-1 receptor. Mol Cell Endocrinol 325:26-35

14. Mentlein R, Gallwitz B, Schmidt WE (1993) Dipeptidyl-peptidase IV hydrolyses gastric inhibitory polypeptide, glucagon-like peptide-1(7-36)amide, peptide histidine methionine and is responsible for their degradation in human serum. Eur J Biochem 214:829-835

15. Deacon CF, Nauck MA, Toft-Nielsen M, Pridal L, Willms B, Holst JJ (1995) Both subcutaneously and intravenously administered glucagon-like peptide I are rapidly degraded from the NH2terminus in type II diabetic patients and in healthy subjects. Diabetes 44:1126-1131

16. Herman GA, Bergman A, Stevens C et al (2006) Effect of single oral doses of sitagliptin, a dipeptidyl peptidase- 4 inhibitor, on incretin and plasma glucose levels after an oral glucose tolerance test in patients with type 2 diabetes. J Clin Endocrinol Metab 91: 4612-4619

17. Rauch T, Graefe-Mody U, Deacon CF et al (2012) Linagliptin increases incretin levels, lowers glucagon, and improves glycemic control in type 2 diabetes mellitus. Diabetes Ther 3:10

18. Landersdorfer CB, He YL, Jusko WJ (2012) Mechanism-based population modelling of the effects of vildagliptin on GLP-1, glucose and insulin in patients with type 2 diabetes. Br J Clin Pharmacol 73:373-390

19. Eto T, Inoue S, Kadowaki T (2012) Effects of once-daily teneligliptin on 24-h blood glucose control and safety in Japanese patients with type 2 diabetes mellitus: a 4-week, randomized, double-blind, placebo-controlled trial. Diabetes Obes Metab 14:1040 1046

20. Nakamura Y, Inagaki M, Shimizu T et al (2013) Long-term effects of alogliptin benzoate in hemodialysis patients with diabetes: a 2-year study. Nephron Clin Pract 123:46-51

21. Yang HK, Min KW, Park SW et al (2015) A randomized, placebocontrolled, double-blind, phase 3 trial to evaluate the efficacy and safety of anagliptin in drug-naïve patients with type 2 diabetes. Endocr J 62:449-462

22. Lambeir AM, Durinx C, Scharpé S, De Meester I (2003) Dipeptidyl-peptidase IV from bench to bedside: an update on structural properties, functions, and clinical aspects of the enzyme DPP IV. Crit Rev Clin Lab Sci 40:209-294 
23. Chen X (2006) Biochemical properties of recombinant prolyl dipeptidases DPP-IV and DPP8. Adv Exp Med Biol 575:27-32

24. Mentlein R, Dahms P, Grandt D, Krüger R (1993) Proteolytic processing of neuropeptide $\mathrm{Y}$ and peptide $\mathrm{YY}$ by dipeptidyl peptidase IV. Regul Pept 49:133-144

25. Kato N, Oka M, Murase T et al (2011) Discovery and pharmacological characterization of $\mathrm{N}-[2-(\{2-[(2 \mathrm{~S})-2$-cyanopyrrolidin-1-yl]2-oxoethyl $\}$ amino) -2-methylpropyl]-2-methyl pyrazolo[1,5-a]pyrimidine-6-carboxamide hydrochloride (anagliptin hydrochloride salt) as a potent and selective DPP-IV inhibitor. Bioorg Med Chem 19:7221-7227

26. Furuta S, Tamura M, Hirooka H, Mizuno Y, Miyoshi M, Furuta Y (2013) Pharmacokinetic disposition of anagliptin, a novel dipeptidyl peptidase-4 inhibitor, in rats and dogs. Eur J Drug Metab Pharmacokinet 38:87-96

27. Deacon CF, Hughes TE, Holst JJ (1998) Dipeptidyl peptidase IV inhibition potentiates the insulinotropic effect of glucagon-like peptide 1 in the anesthetized pig. Diabetes 47:764-769

28. Kubota N, Kubota T, Itoh S et al (2008) Dynamic functional relay between insulin receptor substrate 1 and 2 in hepatic insulin signaling during fasting and feeding. Cell Metab 8:49-64

29. Vincent MA, Clerk LH, Lindner JR et al (2004) Microvascular recruitment is an early insulin effect that regulates skeletal muscle glucose uptake in vivo. Diabetes 53:1418-1423

30. Jansson PA, Fowelin JP, von Schenck HP, Smith UP, Lonnroth PN (1993) Measurement by microdialysis of the insulin concentration in subcutaneous interstitial fluid. Importance of the endothelial barrier for insulin. Diabetes 42:1469-1473

31. Sjostrand M, Gudbjornsdottir S, Holmang A, Lonn L, Strindberg L, Lonnroth P (2002) Delayed transcapillary transport of insulin to muscle interstitial fluid in obese subjects. Diabetes 51:2742-2748
32. Ogihara T, Asano T, Ando $\mathrm{K}$ et al (2001) Insulin resistance with enhanced insulin signaling in high-salt diet-fed rats. Diabetes 50: 573-583

33. De Leon DD, Deng S, Madani R, Ahima RS, Drucker DJ, Stoffers DA (2003) Role of endogenous glucagon-like peptide-1 in islet regeneration after partial pancreatectomy. Diabetes 52:365-371

34. Sunami Y, Sesoko S, Kaku K (2012) Pharmacokinetics and pharmacodynamics of single and multiple doses of anagliptin, a novel inhibitor of dipeptidyl peptidase-4. Jpn Pharmacol Ther 40:847858 [article in Japanese]

35. Panjwani N, Mulvihill EE, Longuet C et al (2013) GLP-1 receptor activation indirectly reduces hepatic lipid accumulation but does not attenuate development of atherosclerosis in diabetic male ApoE(-/-) mice. Endocrinology 154:127-139

36. Matsubara J, Sugiyama S, Sugamura K et al (2012) A dipeptidyl peptidase- 4 inhibitor, des-fluoro-sitagliptin, improves endothelial function and reduces atherosclerotic lesion formation in apolipoprotein E-deficient mice. J Am Coll Cardiol 59:265-276

37. Chai W, Dong Z, Wang N et al (2012) Glucagon-like peptide 1 recruits microvasculature and increases glucose use in muscle via a nitric oxide-dependent mechanism. Diabetes 61:888-896

38. Nakagami H, Pang Z, Shimosato T et al (2014) The dipeptidyl peptidase-4 inhibitor teneligliptin improved endothelial dysfunction and insulin resistance in the SHR/NDmcr-cp rat model of metabolic syndrome. Hypertens Res 37:629-635

39. Dong Z, Chai W, Wang W et al (2013) Protein kinase A mediates glucagon-like peptide 1-induced nitric oxide production and muscle microvascular recruitment. Am J Physiol Endocrinol Metab 304: E222-E228

40. Kusunoki M, Sato D, Nakamura T et al (2015) DPP-4 inhibitor teneligliptin improves insulin resistance and serum lipid profile in Japanese patients with type 2 diabetes. Drug Res 65:532-534 